Disclosure This series has emanated from research supported in part by a grant from Science Foundation Ireland (SFI) and the European Regional Development Fund (ERDF) under grant number 13/RC/2073_P2. It is the independent clinical practice of the authors. Cerenovus provided support for post hoc data collection and analysis.

\section{EP45* BEYOND PROXIMAL LARGE VESSEL OCCLUSIONS: OUTCOME OF MECHANICAL THROMBECTOMY IN DISTAL VESSEL OCCLUSIONS IN THE EXCELLENT REGISTRY - INTERIM ANALYSIS}

${ }^{1}$ OO Zaidat, ${ }^{2}$ RG Nogueira, ${ }^{3}$ AH Siddiqui, ${ }^{4}$ AJ Yoo, ${ }^{5}$ RA Hanel, ${ }^{6}$ W Hacke, ${ }^{7} \mathrm{~T}$ Jovin, ${ }^{8}$ f Fiehler, ${ }^{9} \mathrm{SF}$ De Meyer, ${ }^{10} \mathrm{DS}$ Liebeskind, ${ }^{11} \mathrm{D}$ Haussen, ${ }^{12} \mathrm{~V}$ Inoa, ${ }^{13} \mathrm{~W}$ Humphries, ${ }^{14} \mathrm{~KB}$ Woodward, ${ }^{15} \mathrm{PM}$ Jabbour, ${ }^{16} \mathrm{O}$ Francois, ${ }^{17} \mathrm{El}$ Levy, ${ }^{18} \mathrm{H}$ Bozorgchami, ${ }^{19} \mathrm{~J}$ Cohen, ${ }^{20} \mathrm{~S}$ Boor, ${ }^{21} \mathrm{SR}$ Dashti, ${ }^{22} \mathrm{MA}$ Taqi, ${ }^{23} \mathrm{RF}$ Budzik, ${ }^{24} \mathrm{CM}$ Schirmer, ${ }^{25} \mathrm{MS}$ Hussain, ${ }^{26} \mathrm{~L}$ Estrade, ${ }^{27} \mathrm{RA}$ De Leacy, ${ }^{28} \mathrm{AS}$ Puri, ${ }^{29} \mathrm{R}$ Chitale, ${ }^{30} \mathrm{C}$ Brekenfeld, ${ }^{31,32} \mathrm{~T}$ Andersson. ${ }^{1}$ Neuroscience Department, Mercy St Vincent Medical Center, Toledo, OH; ${ }^{2}$ Department of Neurosurgery, Emory University School of Medicine, Atlanta, GA; ${ }^{3}$ Department of Neurosurgery, SUNY Buffalo, Buffalo, NY; ${ }^{4}$ Neurointervention, Texas Stroke Institute, Plano, $T X ;{ }^{5}$ Department of Neurosurgery, Baptist Stroke and Cerebrovascular Center, Jacksonville, FL, USA; ${ }^{6}$ Department of Neurology, University of Heidelberg, Heidelberg, Germany; ${ }^{7}$ Department of Neurology, University of Pittsburgh Medical Center, Pittsburgh, PA; ${ }^{8}$ Department of Neurosurgeny, University Medical Center Hamburg, Hamburg, GA, USA; ${ }^{9}$ Laboratory for Thrombosis Research, KU Leuven Campus Kulak Kortrijk, Kortrijk, Belgium; ${ }^{10}$ Department of Neurology, University of California Los Angeles, Los Angeles, CA; ${ }^{11}$ Marcus Stroke and Neuroscience Center, Grady Memorial Hospital, Atlanta, GA; ${ }^{12}$ Semmes-Murphey Neurologic and Spine Clinic, Department of Neurosurgery, University of Tennessee Health Science Center, Memphis, TN; ${ }^{13}$ Division of Neurosurgery, Department of Surgery, University of Missouri-Columbia Medical School, Columbia, MO; ${ }^{14}$ Department of Radiology, Fort Sanders Regional Medical Center, Knoxville, $T N_{i}{ }^{15}$ Department of Neurological Surgery, Thomas Jefferson University Hospital, Philadelphia, PA, USA; ${ }^{16}$ Department of Medical Imaging, AZ Groeninge, Kortrijk, Belgium; ${ }^{17}$ Department of Neurosurgery, University at Buffalo, State University of New York, Buffalo, NY; ${ }^{18}$ Department of Neurology, Oregon Health and Sciences, Portland, OR, USA; ${ }^{19}$ Neurosurgery Department, Hadassah University Medical Center, Jerusalem, Israel; ${ }^{20}$ Institute of Neuroradiology, University Medical Center of the Johannes Gutenberg University Mainz, Mainz, Germany; ${ }^{21}$ Department of Neurological Surgery Norton Neuroscience Institute, Norton Healthcare, Louisville, KY; ${ }^{22}$ Department of Neurology and Neurosurgery, Los Robles Hospital and Medical Center, Thousand Oaks, CA; ${ }^{23}$ Department of Radiology, Riverside Methodist Hospital, Columbus, $\mathrm{OH}_{;}{ }^{24}$ Department of Neurosurgery, Geisinger, Danville, PA; ${ }^{25}$ Cerebrovascular Center, Department of Neurology, Neurological Institute, Cleveland Clinic, Cleveland, OH, USA; ${ }^{26}$ Department of Interventional Neuroradiology, Centre Hospitalier Universitaire de Lille, Lille, France; ${ }^{27}$ Department of Neurosurgery, Icahn School of Medicine at Mount Sinai, New York, NY; ${ }^{28}$ Department of Radiology, University of Massachusetts, Worcester, MA; ${ }^{29}$ Department of Neurosurgery, Vanderbilt University Medical Center, Nashville, TN, USA; ${ }^{30}$ Department of Neuroradiology, University Medical Center Hamburg, Hamburg, Germany; ${ }^{31}$ Department of Neuroradiology, Karolinska University Hospital and Clinical Neuroscience Karolinska Institute, Stockholm, Sweden; ${ }^{32}$ Medical Imaging, AZ Groeninge , Kortrijk, Belgium

\subsection{6/neurintsurg-2021-ESMINT.44}

Introduction EXCELLENT (NCT03685578) is a prospective, single-arm, multicenter, real-world, international registry of mechanical thrombectomy (MT) with EmboTrap as first-line device. There is increasing interest in applying MT to acute distal vessel occlusion (DiVO), with limited data. This interim analysis evaluates the angiographic, clinical and safety outcomes in DiVO.

Aim of the Study To compare MT outcomes in Medium Vessel occlusion (MeVO) vs DiVO.

Methods MeVO was defined as proximal M2 and DiVO as distal M2, M3, P1, P2, and A2 occlusions. Independent core lab adjudicated occlusion location, reperfusion, and intracranial hemorrhage (ICH). 90-day mRS was completed by independent site investigators blinded to procedural data.
Results 64/532(12.0\%) patients had MeVO, and 44/532(8.3\%) had DiVO (37 M2, 4 P2, 1 A2, 1 P1, 1 M3). Final mTICI2b-3 was $87.1 \%(54 / 62)$ in $\mathrm{MeVO}$ vs $84.1 \%(37 / 44)$ in DiVO. First Pass (FP) mTICI2c was $17.5 \%(11 / 63)$ in $\mathrm{MeVO}$ vs $6.8 \%(3 / 44)$ in DiVO; while the two groups had nearly identical FP-mTICI3 (20.6\% vs 20.5\%). 90-day mRS $0-2$ or equal to pre-stroke was achieved in 55.4\%(31/56) $\mathrm{MeVO}$ and in $45.9 \%(17 / 37)$ DiVO patients. All-cause 90-day mortality was $22.4 \%(13 / 58)$ in $\mathrm{MeVO}$ and $21.4 \%(9 / 42)$ in DiVO. The rate of any $\mathrm{ICH}$ was $50.8 \%(31 / 61)$ in $\mathrm{MeVO}$ vs $37.5 \%(15 /$ $40)$ in DiVO, while subarachnoid hemorrhage was $27.9 \%(17 /$ 61) and 22.5\%(9/40) in MeVO and DiVO, respectively.

Conclusions In this interim analysis, patients with DiVO presenting with acute ischemic stroke and undergoing MT had rates of revascularization and clinical and safety outcomes comparable to MeVO. If confirmed in the full dataset, these findings can inform MT case selection.

Disclosure EXCELLENT is sponsored by Cerenovus. Dr. Zaidat serves as a consultant for Neuravi, Stryker, Penumbra, and Medtronic.

\section{EP46* FIRST PASS EFFECT AND ASSOCIATED CLOT CHARACTERISTICS IN THE EXCELLENT REGISTRY - INTERIM ANALYSIS}

${ }^{1} \mathrm{~T}$ Jovin, ${ }^{2} \mathrm{RG}$ Nogueira, ${ }^{3} \mathrm{AH}$ Siddiqui, ${ }^{4} \mathrm{AJ}$ Yoo, ${ }^{5} \mathrm{RA}$ Hanel, ${ }^{6} \mathrm{OO}$ Zaidat, ${ }^{7} \mathrm{~W}$ Hacke, ${ }^{8} \mathrm{~J}$ Fiehler, ${ }^{9} \mathrm{SF}$ De Meyer, ${ }^{10} \mathrm{~W}$ Brinjikji,, ${ }^{11} \mathrm{~K}$ Doyle, ${ }^{12} \mathrm{DS}$ Liebeskind, ${ }^{13} \mathrm{D}$ Haussen, ${ }^{14} \mathrm{~V}$ Inoa, ${ }^{15} \mathrm{~W}$ Humphries, ${ }^{16} \mathrm{~KB}$ Woodward, ${ }^{17} \mathrm{PM}$ Jabbour, ${ }^{18} \mathrm{O}$ Francois, ${ }^{19} \mathrm{El}$ Lewy, ${ }^{20} \mathrm{H}$ Bozorgchami, ${ }^{21} \mathrm{~J}$ Cohen, ${ }^{22} \mathrm{~S}$ Boor, ${ }^{23} \mathrm{SR}$ Dashti, ${ }^{24} \mathrm{MA}$ Taqi, ${ }^{25} \mathrm{RF}$ Budzik, ${ }^{26} \mathrm{CM}$ Schirmer, ${ }^{27} \mathrm{MS}$ Hussain, ${ }^{28}$ L Estrade, ${ }^{29}$ RA De Leacy, ${ }^{30}$ AS Puri, ${ }^{31} \mathrm{R}$ Chitale, ${ }^{32} \mathrm{C}$ Brekenfeld, ${ }^{18,33} \mathrm{~T}$ Andersson. ${ }^{1}$ Cooper Neurological Institute, Cooper University Hospital, Camden, NJ; ${ }^{2}$ Department of Neurosurgery, Emory University School of Medicine, Atlanta, GA; ${ }^{3}$ Department of Neurosurgery, SUNY Buffalo, Buffalo, NY; ${ }^{4}$ Neurointervention, Texas Stroke Institute, Plano, TX; ${ }^{5}$ Department of Neurosurgery, Baptist Stroke and Cerebrovascular Center, Jacksonville, FL; ${ }^{6}$ Department of Neuroscience, Mercy St Vincent Medical Center, Toledo, OH, USA; ${ }^{7}$ Department of Neurology, University of Heidelberg, University of Heidelberg; ${ }^{8}$ Department of Neurosurgery, University Medical Center Hamburg, Hamburg, Germany; ${ }^{9}$ Laboratory for Thrombosis Research, KU Leuven, Campus Kulak Kortrijk, Kortrijk, Belgium; ${ }^{10}$ Department of Radiology, Mayo Clinic, Rochester, MN, USA; ${ }^{11}$ Department of Physiology, National University of Ireland, Galway, Galway, Ireland; ${ }^{12}$ Department of Neurology, University of California Los Angeles, Los Angeles, CA; ${ }^{13}$ Marcus Stroke and Neuroscience Center, Grady Memorial Hospital, Atlanta, GA; ${ }^{14}$ Semmes-Murphey Neurologic and Spine Clinic, Department of Neurosurgery, University of Tennessee Health Science Center, Memphis, TN; ${ }^{15}$ Division of Neurosurgery, Department of Surgeny, University of Missouri-Columbia Medical School, Columbia, MO; ${ }^{16}$ Department of Radiology, Fort Sanders Regional Medical Center, Knoxville, $T N_{;}{ }^{17}$ Department of Neurological Surgery, Thomas Jefferson University Hospital, Philadelphia, PA, USA; ${ }^{18}$ Department of Medical Imaging, AZ Groeninge, Kortrijk, Belgium; ${ }^{19}$ Department of Neurosurgery, University at Buffalo, State University of New York, Buffalo, NY; ${ }^{20}$ Department of Neurology, Oregon Health and Sciences, Portland, OR, USA; ${ }^{21}$ Neurosurgery Department, Hadassah University Medical Center, Jerusalem, Israel; ${ }^{22}$ Institute of Neuroradiology, University Medical Center of the Johannes Gutenberg University Mainz, Mainz, Germany; ${ }^{23}$ Department of Neurological Surgery Norton Neuroscience Institute, Norton Healthcare, Louisville, KY; ${ }^{24}$ Department of Neurology and Neurosurgery, Los Robles Hospital and Medical Center, Thousand Oaks, CA; ${ }^{25}$ Department of Radiology, Riverside Methodist Hospital, Columbus, $\mathrm{OH}_{;}{ }^{26}$ Department of Neurosurgery, Geisinger, Danville, $P A ;{ }^{27}$ Cerebrovascular Center, Department of Neurology, Neurological Institute, Cleveland Clinic, Cleveland, OH, USA; ${ }^{28}$ Department of Interventional Neuroradiology, Centre Hospitalier Universitaire de Lille, Lille, France; ${ }^{29}$ Department of Neurosurgery, Icahn School of Medicine at Mount Sinai, New York, NY; ${ }^{30}$ Department of Radiology, University of Massachusetts, Worcester, MA; ${ }^{31}$ Department of Neurosurgery, Vanderbilt University Medical Center, Nashville, TN, USA; ${ }^{32}$ Department of Diagnostic and Interventional Neuroradiology, University Medical Center Hamburg, Hamburg, Germany; ${ }^{33}$ Department of Neuroradiology, Karolinska University Hospital and Clinical Neuroscience Karolinska Institute, Stockholm, Sweden

10.1136/neurintsurg-2021-ESMINT.45 